\title{
The Long Shadow of Peers: Adolescent Networks and Young Adult Mental Health
}

\author{
Molly Copeland if
}

\section{check for}

updates

Citation: Copeland, Molly. 2021. The Long Shadow of Peers: Adolescent Networks and Young Adult Mental Health. Social Sciences 10: 231. https://doi.org/10.3390/socsci10060231

Academic Editor: Robin Gauthier

Received: 26 April 2021

Accepted: 12 June 2021

Published: 17 June 2021

Publisher's Note: MDPI stays neutral with regard to jurisdictional claims in published maps and institutional affiliations.

Copyright: (C) 2021 by the author. Licensee MDPI, Basel, Switzerland. This article is an open access article distributed under the terms and conditions of the Creative Commons Attribution (CC BY) license (https:/ / creativecommons.org/licenses/by/ $4.0 /)$.
Department of Sociology, Michigan State University, East Lansing, MI 48824, USA; mcope@msu.edu

\begin{abstract}
Connections with peers play an important role in adolescent mental health, but their lasting impact is unclear. This study examines whether structural status and support in adolescent networks predict depressive symptoms years later. Using data from the PROSPER Peers study $(n=1017)$, I find that the persistent effects of networks differ based on the mental health of teens and their friends. Structures of status and support relate to young adult mental health only for individuals who experience depressive symptoms as teens. Among depressive adolescents, popularity predicts lower subsequent depressive symptoms, while high prestige predicts higher depressive symptoms in young adulthood. Embeddedness among depressed friends also predicts higher young adult depressive symptoms. Overall, findings suggest relationships with peers can set the stage for mental health for adolescents who experience depressive symptoms or have depressive friends.
\end{abstract}

Keywords: social networks; adolescence; peer networks; mental health; young adulthood

\section{Introduction}

Social relationships are critical to mental health (Durkheim [1897] 2006; Cohen 2004), and for adolescents in the United States, peer relationships affect health and health behaviors (Valente 2010; Umberson et al. 2010). Positions of how individuals are situated among interconnected friendships of teens in school, or the peer social network, can promote teens' mental health by conveying social status and social support (Millings et al. 2012; Ueno 2005; Cornwell 2003). Moreover, any association of adolescent mental distress with structural status and support depends in part on the mental health of youth and those to whom they are connected (Cheadle and Goosby 2012).

Yet, despite the wealth of research on adolescent peer relationships, whether or not peer networks have a lasting impact on mental health after adolescence is unclear. Adolescent depressive symptoms are tightly linked to those in young adulthood, a period of substantial transition, upheaval, and change (Allen et al. 2014; Edgerton et al. 2018). Adolescents' peer experiences may affect mental health or psychosocial skills to have a persistent effect on young adult mental health (Costello et al. 2008). Alternatively, the developmental importance of peers may be limited to adolescence, with any impact waning as teens transition into young adult environments. Understanding how connections with peers in the sensitive period of adolescence relate to young adult well-being can clarify the importance of peers for long-term mental health.

\subsection{Adolescent Peer Networks and Mental Health}

Adolescence is a sensitive period of the life course for social development when connections with same-age peers become focal relationships that guide identity, behaviors, and mental health (Cotterell 2007; Johnson et al. 2011; Umberson et al. 2010). Patterns of social relationships among school peers create a web of social network connections that has a characterizable structure, and a teen's position in that structure relates to mental well-being (Valente 2010; Cotterell 2007).

Two main ways that peer networks relate to mental health are by providing the underlying structural conditions for status ${ }^{1}$ and support. Social status stems from connections across 
the whole peer network (Reynolds and Crea 2015). Highly visible, central, well-connected positions such as high popularity among other highly popular peers can convey social status that fosters mental well-being through greater self-esteem, a sense of belonging, or positive self-concept (Ueno 2005; Millings et al. 2012; Kornienko and Santos 2014). Unlike social status, structures of social support do not depend on the wider network context, but rather on the network structure among close friends, as support is typically provided in close relationships and shaped by factors such as whether or not friends are also friends with each other (Thoits 2011; Falci and McNeely 2009). In adolescence, peers are an important source of social support and bolster psychosocial resources, buffer against stress, and predict decreased depression (Ohannessian et al. 2018; Costello et al. 2008; Cornwell 2003).

However, greater peer connection does not always benefit mental health. While lacking connections to peers predicts higher levels of depressive symptoms (Ueno 2005), having too many peer ties also predicts depression (Falci and McNeely 2009). For example, some social status among peers is beneficial, but having very high status increases stress, scrutiny, and over-regulation of behavior, to the detriment of adolescent mental health (Reynolds and Crea 2015; Falci and McNeely 2009). Similarly, close friendships that are typically supportive may spur co-rumination, increasing mental distress (Rose et al. 2014).

One factor that might distinguish when peer networks predict better or worse mental health is the mental distress of who is being connected in the network. For example, for teens already experiencing anxiety and mental distress, high social status increases feelings of scrutiny or judgment, which exacerbate depressive symptoms (Kornienko and Santos 2014). Depressive teens may induce conflict, demand greater support, or instigate co-rumination with close friends in ways that strain relationships and subsequent mental health (Rose et al. 2014).

Peers' mental health may also affect how network structures of status and support relate to depressive levels. High-status positions predict increased depressive levels when friends are depressive (Reynolds and Crea 2015), and individuals who are not themselves experiencing depressive symptoms but have depressive friends may receive less social support from those friends or become engaged in co-rumination to the detriment of their own mental health (Schaefer et al. 2011; Rose et al. 2014).

In this way, how peer network structures of social status and structural support relate to mental health may depend upon the depressive levels of adolescents and friends. However, it is unclear whether such moderating effects persists across life course transitions to affect young adult mental health.

\subsection{Adolescent Peers and Young Adult Mental Health}

Experiences of status or support among peers in the sensitive period of adolescence may have consequences for subsequent life course stages. Status and support in adolescence affect social skills, coping skills, and other psychosocial traits that youth carry forward into adulthood to affect adult mental health (Costello et al. 2008; Segrin and Rynes 2009). For example, perceived family social support in adolescence predicts lower depressive levels at the stressful transition into young adulthood (Edgerton et al. 2018). Respondents self-reporting higher perceived peer social support (rather than peer network structural measures used here) experience lower depressive levels in young adulthood, while conflict predicts higher subsequent depression (Gore and Aseltine 2003). Peer relationship quality similarly predicts later relationship quality (Cook et al. 2016). Popularity in the peer network as a teen also predicts depressive levels for young women across adolescence and into adulthood (Kamis and Copeland 2020). While these patterns suggest that experiences among adolescent peers can have lasting effects on mental health beyond adolescence, the developmental salience of peers may be limited to adolescence, so that associations between mental health and teen network position do not persist as youth age into the new roles, environments, and experiences of young adulthood. Regardless, how any association between teen networks and young adult mental health may differ for network structures of status and support and with the mental health of teens and their friends remains unclear, warranting consideration in the current study. Research questions here examine whether: 
- Adolescent peer network structures of status and support predict young adult mental health;

- Adolescents' and friends' depressive symptoms moderate any associations between teen social networks and depressive levels in young adulthood.

\section{Data and Methods}

\subsection{Data}

Data for this study were collected in the PROSPER (PROmoting School-communityuniversity Partnerships to Enhance Resilience) Project, a randomized controlled trial evaluating a partnership-based delivery system for evidence-based preventive interventions (Spoth et al. 2004, 2013). This study utilizes data from the in-school survey portion of the PROSPER project and uses social network measures developed from this survey as part of PROSPER Peers. Surveyed schools are located in rural and semi-rural Iowa and Pennsylvania in the United States and have predominantly white student populations. Sampled districts were randomly selected from the pool of districts meeting parameters of interest in the larger PROSPER intervention, with enrollments between 1500 and 5200 and with at least $15 \%$ of students in each district from low-income families. Surveys were administered to students during school hours starting in the fall of sixth grade (approximately ages 11-12) in two cohorts of students in successive calendar years in 2002 and 2003. Subsequent surveys were administered every spring through grade 12 (approximately age 18). After high school, a follow-up survey of 1985 respondents at age 19 was completed via computer-assisted telephone interview or web-based survey. A further subset of 1628 completed a second wave of young adult survey at age 22. Data collection met ethical requirements, per institutional boards of managing institutions. Further details of the study design are described in prior work (Spoth et al. 2013).

In the current study, outcome measures come from both waves of the young adult follow-up surveys. Adolescent measures come from waves 5-8 of the in-school surveys, as these waves are when variables of interest are assessed. In these waves, students are in grades 9-12, in 2006-2010. The average survey response rate was $81 \%$.

Data on adolescent social networks come from the friendship nomination section of the in-school adolescent survey. Students wrote the names of up to seven best or close friends in their school and grade. Nominations were then matched to peer surveys (match rate $=79 \%$ ), linking survey responses. This design creates sociocentric or whole network friendship networks where ties are matched across respondents in a setting (rather than reflecting only one focal individual's perspective) for a grade in school at each wave, enabling examination of structural patterns across the whole network. Network data were thus collected during adolescence rather than retrospectively assessed or measured as perceptions of status or support, which may be subject to additional bias for those experiencing depressive symptoms (McGrath and Repetti 2002). The rich adolescent peer network data and mental health survey of the same respondents in young adulthood means this dataset provides a unique opportunity to assess long-term mental health consequences of teen networks.

\subsection{Dependent Variable}

The outcome variable of depressive symptoms is an average depression score drawn from 14 items from the CES-D (Radloff 1977, $\alpha=0.87$ ). Responses ranging from 0 to 3 for each item were summed in each young adulthood wave and then averaged to create one score of depressive symptoms for young adulthood that could range from 0 to 42 . Alternative specifications, detailed in the Robustness section, yielded similar results. Descriptive statistics for all measures are in Table 1. 
Table 1. Descriptive statistics of analytic sample in PROSPER Peers.

\begin{tabular}{ccccc}
\hline & Mean or Proportion & (SD) & Min & Max \\
\hline Depressive Symptoms in & 7.15 & $(6.02)$ & 0 & 37.5 \\
Young Adulthood & 0.43 & - & 0 & 1 \\
Male & 0.93 & - & 0 & 1 \\
White & & & & \\
\hline Adolescent Networks & 3.07 & $(2.13)$ & 0 & 12 \\
\hline Popularity & 0.87 & $(0.59)$ & 0 & 3.95 \\
Prestige & 0.53 & $(0.32)$ & 0 & 1 \\
Reciprocity & 0.23 & $(0.20)$ & 0 & 1 \\
Cohesion & & & & \\
\hline Adolescence Controls & 0.22 & $(0.41)$ & 0 & 1 \\
\hline HS Depression & 0.14 & $(0.17)$ & 0 & 1 \\
HS Friends' Depression & 3.19 & $(1.80)$ & 0 & 7 \\
Gregariousness & 9.3 & $(5.48)$ & 0 & $20+$ \\
Out-of-grade Friends & -0.14 & $(0.36)$ & -1.15 & 0.77 \\
Family Relations & 0.13 & - & 0 & 1 \\
Low-SES & 0.50 & - & 0 & 1 \\
RCT Treatment & & & & \\
\hline Young Adulthood Controls & 0.36 & $(0.48)$ & 0 & 1 \\
\hline Parental Home & 0.82 & $(0.38)$ & 0 & 1 \\
Maintain Friend Contact & 0.68 & $(0.47)$ & 0 & 1 \\
Full-time Job/Student & 2.26 & $(0.89)$ & 1 & 5 \\
Physical Health & & & & \\
Observations & & & \\
\hline
\end{tabular}

\subsection{Network Variables}

Two social network variables capture status. Measures are averaged across adolescence to create a general social profile of teens' adolescent networks, as teens' social positions are generally stable year to year (Moody et al. 2011). Alternative specifications, discussed in the Robustness section, yield similar results, but worse model fit.

Popularity is measured by average in-degree, a count of how many friendship nominations an adolescent receives from peers (Valente 2010). Another measure capturing broader patterns of status across the entire network is prestige, measured by average Bonacich centrality (Bonacich 1987). This measure takes into account the popularity of an adolescent's friends: high values indicate teens who are popular among popular peers, while lower values indicate unpopular teens or teens who are popular only among unpopular peers. Both popularity and prestige as measured here are commonly used to assess peer network social status (e.g., Reynolds and Crea 2015; Ueno 2005).

Two social network measures assess structural support. Reciprocity is an average across adolescence of an adolescent's proportion of sent and received ties that are mutual (where an adolescent names a peer as a friend and that peer also names them in turn, Valente 2010). Reciprocity measures the proportion of an adolescent's ties that are stronger, mutually agreed upon friendships. Cohesion is measured by average ego-network density, or the proportion of an adolescent's friends who are also friends with each other (Valente 2010). High cohesion indicates tight-knit friend groups.

\subsection{Demographics and Control Variables}

Several measures adjust for characteristics of respondents during adolescence. HS depression indicates an individual's experience of depressive symptoms in high school, measured by a binary yes/no item drawn from the CES-D. This measure is assessed in the last two years of high school in the survey, asking "In the past 12 months: Did you ever feel so sad or hopeless almost every day for two weeks or more in a row that you stopped doing some usual activities?". A response of "yes" to that question in either wave 
is recorded as a 1 for the measure. The same survey question answered by each network friend was used to calculate the average of depressive symptoms for each individual's sent and received ties, as friends' depression. Gregariousness is measured by average out-degree or the number of friendship nominations an adolescent sends to school peers (Valente 2010). While gregariousness does not conceptually indicate status (a low-status teen could send many unreciprocated nominations), including gregariousness enables analysis of status (popularity and prestige) net of any association of sent and received ties. Out-of-grade friends is an average of the count variable of an adolescent's number of friends outside of their grade or school at each high school wave. This variable helps to adjust for the restriction of network data to the school-grade boundary to account for youth who may be spending their social energy elsewhere. RCT Treatment is a binary indicator if an adolescent's school district was in the treatment or control group of the larger PROSPER intervention that targeted teen resilience against substance use.

Other measures assessed in adolescence adjust for factors that could relate to networks and mental health. Low-SES indicates low socioeconomic status via eligibility for free/reduced-price lunch from a family income under $150 \%$ of the federal poverty line. Family relations addresses teens' relationships with parents, measured by an average of scores on a composite scale of parent-child affective qualities, activities, and reasoning $(\alpha=0.95)$. Male and white measure gender and race and are time-invariant for respondents in this sample.

Other measures adjust for factors in young adulthood that could affect mental health. These variables include averages of binary measures indicating if individuals still live with their parents, as parental home (yes =1), and if individuals still maintain contact with their high school friends, as maintain friend contact (yes =1). An average of a binary measure indicating if an individual is employed full-time or a full-time student, full-time job/student (yes =1), is also included. Physical health is an average of an ordinal measure of self-rated physical health, where higher values indicate worse health, as poor physical health can affect mental health.

\subsection{Analyses}

Models use ordinary least-squares regression (OLS) to examine if adolescent network variables indicating status and structural support predict depressive symptoms in young adulthood. Robust standard errors are used to adjust for heteroskedasticity and provide more conservative estimates of statistical significance (Wooldridge 2012). Students who repeat a grade in the high school portion of the survey are dropped $(n=24)$, as they appear twice in the same wave in both cohorts. Given the relative lack of validated alternative methods of addressing missingness in sociocentric social network measures and the robustness of network measures to missingness (Smith et al. 2017), listwise deletion is used. Deletion of incomplete cases occurs after sociocentric network construction, so that while individual non-response (e.g., an adolescent who declined to take the survey) can still yield missing network data, major concerns of listwise deletion spurring a cascade of missing actors and ties throughout the network or tie missingness driven by link or snowball tracing techniques are not of concern here, though future work should consider these research questions with more advanced network imputation methods (see Krause et al. 2020 for more information on missingness strategies for network data).

Of the young adults completing at least one wave of the survey during young adulthood ( $n=1985), 1256$ respondents have information for both depressive symptoms as a young adult and adolescent social networks. Listwise deletion of cases missing on covariates yields a final analytic sample of $n=1017$.

Model 1 examines the relationship between teen network status and structural support and young adult depressive symptoms net of controls, including depressive levels in high school. Model 2 adds interactions between teen depression and network variables to test if the association of between networks and later mental distress varies for depressive and 
non-depressive youth. Then, Model 3 then examines the role of friends' depression by interacting network measures and friends' average depression.

\section{Results}

Descriptive statistics are shown in Table 1. The average depressive symptoms in young adulthood is about 7 , with an observed maximum of 37.5 out of a potential score of 42 , and $22 \%$ of adolescents report depressive symptoms as teens. These measures indicate distributions of depressive symptoms similar to those typically found in adolescent and young adult samples (Hankin 2006). Averages for the network variables indicating status and structural support align with previous research (Falci and McNeely 2009; Ueno 2005). Average popularity as an adolescent is around 3, meaning that on average adolescents are named as a friend by 3 peers each year of high school, though some teens are nominated by an average of 12 peers. Prestige is similarly distributed, with an average prestige score of 0.87 , though some adolescents reach nearly 4 . Average reciprocity is 0.53 , indicating that on average slightly over half of the friendship ties observed are reciprocated. The average cohesion in the sample is 0.23 , meaning that about $23 \%$ of all possible friendship ties exist between adolescents' friends.

Results of analyses are shown in Table 2. Model 1 indicates that worse family relations, depressive symptoms as an adolescent, and worse physical health in young adulthood predict higher depressive symptoms as a young adult. In Model 1, status and structural support in adolescent networks are not significantly associated with young adult depressive symptoms.

Table 2. OLS regression of depressive symptoms in young adulthood on adolescent social network status and support in PROSPER Peers ${ }^{\text {a }}$.

\begin{tabular}{|c|c|c|c|c|c|c|}
\hline & \multicolumn{2}{|c|}{ Model 1} & \multicolumn{2}{|c|}{ Model 2} & \multicolumn{2}{|c|}{ Model 3} \\
\hline & $\beta$ & (SE) & $\beta$ & (SE) & $\beta$ & (SE) \\
\hline Male & 0.32 & $(0.37)$ & 0.38 & $(0.37)$ & 0.31 & $(0.37)$ \\
\hline White & -0.75 & $(0.72)$ & -0.56 & $(0.74)$ & -0.72 & $(0.73)$ \\
\hline \multicolumn{7}{|l|}{ Status } \\
\hline Popularity & 0.02 & $(0.16)$ & 0.22 & $(0.16)$ & -0.10 & $(0.20)$ \\
\hline Prestige & -0.55 & $(0.59)$ & -1.10 & $(0.60)$ & -0.02 & $(0.68)$ \\
\hline \multicolumn{7}{|l|}{ Structural Support } \\
\hline Reciprocity & 0.34 & $(0.78)$ & -0.14 & $(0.82)$ & 1.12 & $(0.92)$ \\
\hline Cohesion & 0.88 & $(0.89)$ & -0.21 & $(0.90)$ & -0.90 & (1.01) \\
\hline Popularity X HS Depressi & & & $-1.09 *$ & $(0.46)$ & & \\
\hline Prestige X HS Depression & & & $3.50 *$ & $(1.72)$ & & \\
\hline Reciprocity X HS Depress & & & 2.19 & $(1.87)$ & & \\
\hline Cohesion X HS Depressio & & & 4.55 & $(2.41)$ & & \\
\hline Popularity X Friends Dep & ssion & & & & 0.75 & $(0.98)$ \\
\hline Prestige X Friends Depres & & & & & -4.07 & $(3.29)$ \\
\hline Reciprocity X Friends De & ssion & & & & -4.54 & $(3.20)$ \\
\hline Cohesion X Friends Depr & sion & & & & $13.31^{* *}$ & $(5.06)$ \\
\hline \multicolumn{7}{|c|}{ Adolescent Controls } \\
\hline HS Depression & $2.48 * * *$ & $(0.52)$ & 0.82 & $(1.10)$ & $2.49^{* * *}$ & $(0.53)$ \\
\hline HS Friends' Depression & 2.02 & (1.03) & 2.02 & $(1.04)$ & 2.68 & $(2.39)$ \\
\hline Family Relations & $-2.86^{* * *}$ & $(0.56)$ & $-2.81 * * *$ & $(0.56)$ & $-2.86^{* * *}$ & $(0.56)$ \\
\hline Low-SES & 0.65 & $(0.59)$ & 0.68 & $(0.58)$ & 0.65 & $(0.59)$ \\
\hline Gregariousness & -0.02 & $(0.14)$ & -0.02 & $(0.14)$ & -0.03 & $(0.14)$ \\
\hline Out-of-grade Friends & 0.002 & $(0.03)$ & -0.001 & $(0.03)$ & 0.005 & $(0.03)$ \\
\hline RCT Treatment & 0.57 & $(0.35)$ & 0.57 & $(0.35)$ & 0.56 & $(0.35)$ \\
\hline \multicolumn{7}{|l|}{ Young Adult Controls } \\
\hline Parental Home & 0.73 & $(0.38)$ & 0.66 & $(0.38)$ & 0.71 & $(0.38)$ \\
\hline Maintain Friend Contact & -0.22 & $(0.48)$ & -0.17 & $(0.47)$ & -0.20 & $(0.48)$ \\
\hline Full-time Job/Student & 0.50 & $(0.40)$ & 0.45 & $(0.40)$ & 0.53 & $(0.40)$ \\
\hline Physical Health & $1.90^{* * *}$ & $(0.22)$ & $1.92^{* * *}$ & $(0.22)$ & $1.91^{* * *}$ & $(0.22)$ \\
\hline Intercept & 1.48 & $(1.09)$ & 1.69 & $(1.09)$ & 1.30 & $(1.12)$ \\
\hline Observations & 1017 & & 1017 & & 1017 & \\
\hline AIC & 6336.7 & & 6331.2 & & 6336.2 & \\
\hline
\end{tabular}


However, in Model 2, when network positions are interacted with high school depressive symptoms, the interacted status measures do significantly predict young adult mental health. Popularity predicts reduced depressive symptoms in young adulthood for those who experienced depressive symptoms as teens. This result means that adolescents experiencing depressive symptoms who were popular have lower depressive symptoms in young adulthood than those who were similarly depressive but unpopular. Put another way, low social status presents risks to later mental health for depressive teens. This pattern is shown in Figure 1A. Higher popularity has no significant relationship with the predicted probability of young adult depressive symptoms for non-depressive teens, but for distressed teens, higher popularity predicts lower young adult depressive symptoms.
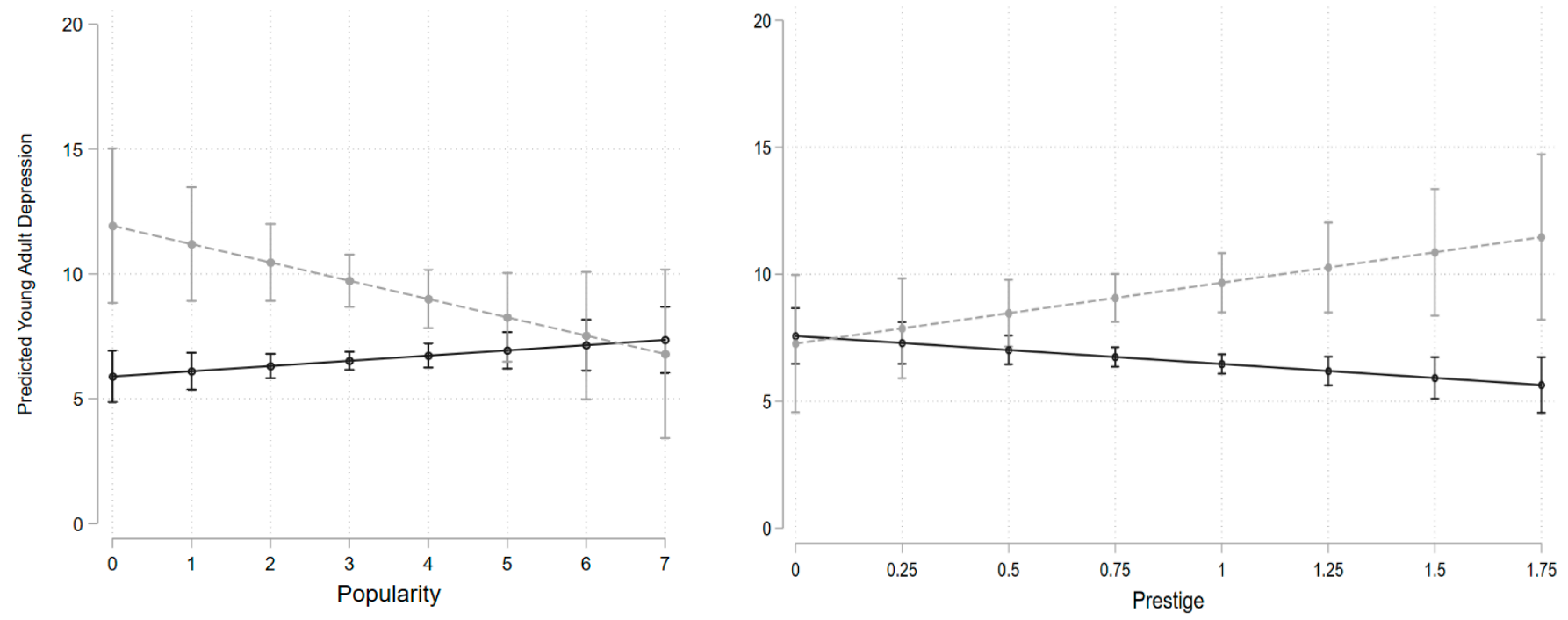

- Non-Depressive in Adolescence

- - Depressive in Adolescence

a Network measures are shown to 95th percentile rather than full range to be conservative. Confidence intervals are at $95 \%$.

(A)

Figure 1. Predicted probability of young adulthood depressive symptoms on adolescent popularity (A) and prestige (B) by adolescent high school depressive symptoms ${ }^{a}$.

Unlike popularity, higher prestige does not benefit later mental health. Greater prestige as an adolescent predicts higher young adult depressive symptoms for depressive teens, shown in Figure 1B. Taken together, these results suggest that for depressive adolescents, having some peers name you as a friend predicts lower subsequent depressive symptoms, but greater status beyond that, such as being highly connected among popular peers, predicts higher depressive symptoms. Measures of structural support are again not associated with young adult depressive symptoms in Model 2.

Model 3 examines if the association between network status and structural support varies with friends' mental distress. Here, measures of status do not relate to later depressive symptoms, but one measure of structural support does. Friends' depression in high school significantly moderates the association between cohesion and later depressive symptoms, shown in Figure 2. Adolescents who are highly cohesively integrated among depressive friends show higher depressive symptoms in young adulthood. Overall, results indicate that networks predict depressive symptoms in young adulthood for depressive teens or those embedded among depressive peers. 


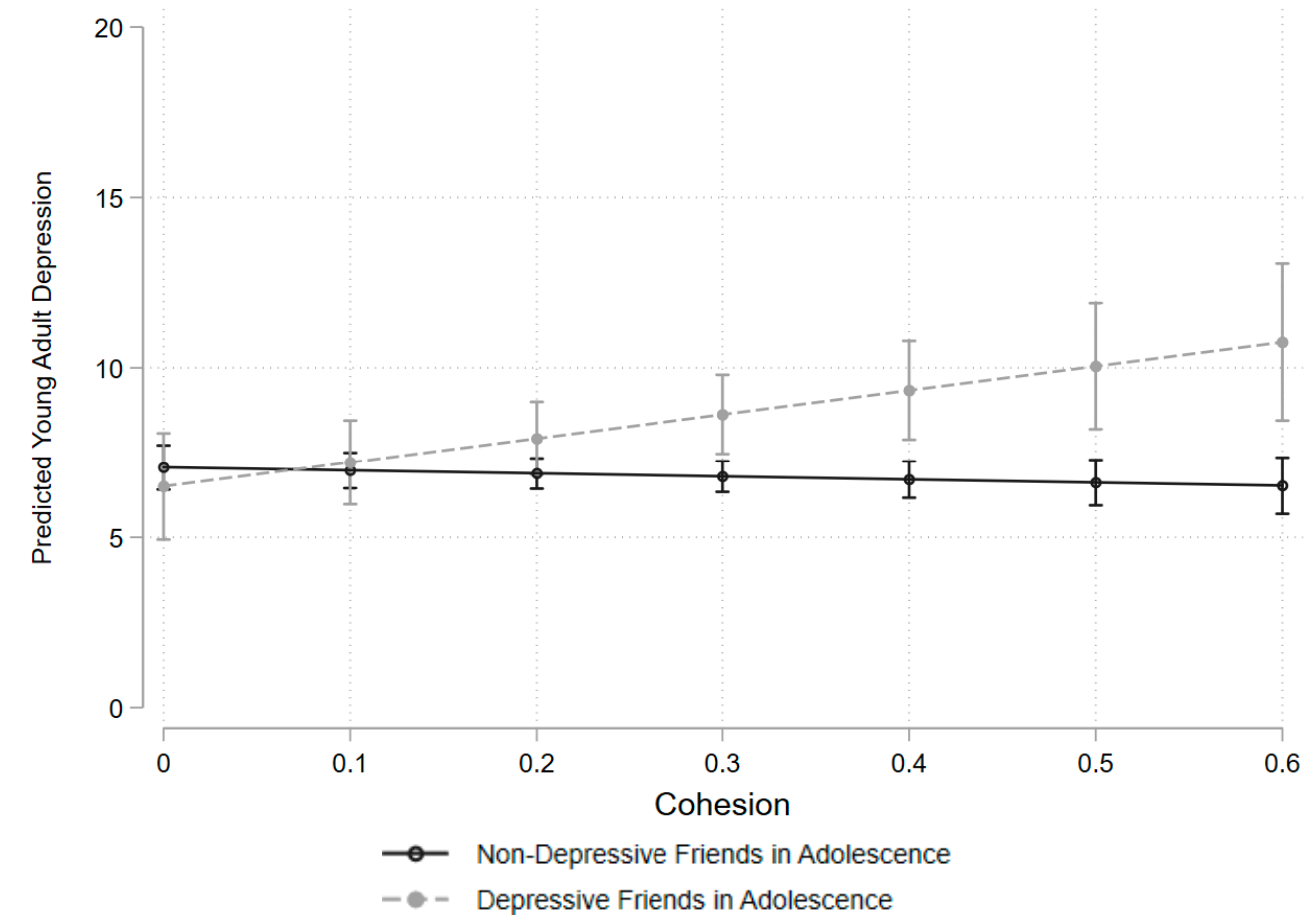

a Network measures are shown to 95th percentile rather than full range to be conservative. Confidence intervals are at $95 \%$.

Figure 2. Predicted probability of young adulthood depressive symptoms on adolescent cohesion by high school friends' depressive symptoms ${ }^{\text {a }}$.

\section{Robustness}

Results shown here are robust to several additions and model specifications. Alternative modeling strategies, including measuring individuals' trajectories of change in each network variable across adolescence, using only adolescent data from the last two years of high school for closer proximity to young adulthood, and using only network measures from the final year of high school show consistent results with those shown here. The models shown here provide the best model fit according to Akaike's Information Critieria (AIC) and preserve the most cases, enabling more reliable analyses of interactions and complex network measures. A fourth model combining both individuals' and friends' depressive symptoms interacted with network measures (available upon request) shows the same pattern of results as models here, which are shown to ease interpretation.

Curvilinear effects of network variables were tested, but worsened model fit, though the curvilinear effect for cohesion was significant in the baseline model. The pattern of results is the same with other specifications of the dependent variable, including using the average (rather than count) of scale items and the maximum depression score from either wave in young adulthood. The operationalization of the outcome shown here aligns with prior work using a summative score for depressive symptoms for interpretability. Log-transforming popularity and prestige yield the same pattern of results, so the nontransformed measures are used here for ease of interpretation. Results are not sensitive to outliers. Models using mean-centered network variables to mitigate multicollinearity (available upon request) yield variance inflation factor values under 10 and yielded the same results, indicating that multicollinearity does not affect results of interest. Noncentered measures are used here for interpretability.

\section{Discussion}

Peer social networks play a vital role in healthy social development in the sensitive period of adolescence (Cotterell 2007; Johnson et al. 2011). Structures of status and support in peer networks may relate to mental health beyond adolescence, as these features foster 
psychosocial traits and social skills that may persist to shape long-term mental health (Cook et al. 2016; Ohannessian et al. 2018). Additionally, the way in which social network positions relate to later mental health likely differs for youth experiencing depressive symptoms or who are connected to depressive peers (Reynolds and Crea 2015). This study uses data spanning adolescence and young adulthood that include rich measures of social network position in adolescence to examine how adolescent network structures of status and structural support predict young adult depressive symptoms in ways that differ with adolescent mental health.

Results indicate multiple ways social network positions relate to young adult mental health. First, peer network status interacts with teens' depressive symptoms. For depressive teens, some status is beneficial, but too much status becomes detrimental. Depressive adolescents who are unpopular have higher levels of subsequent depressive symptoms, while similarly depressive teens who are popular among peers show lower depressive levels as young adults. This result suggests that depressive adolescents may be more vulnerable to negative consequences of low social status among peers, or that popularity, even when experienced concurrently with depressive symptoms, may generate skills or psychosocial benefits that affect later mental health.

However, being in a high prestige position, meaning being highly popular among other highly popular peers, predicts higher young adult depressive symptoms for those who were depressive as teens. These prestigious network positions may generate scrutiny or social demands that increase depressive symptoms for depressive youth, or depressive teens with high prestige might experience greater stress from trying to maintain their high status (Falci and McNeely 2009; Reynolds and Crea 2015). Together, these results align with prior work that suggests that teens' mental health benefits from having some friends but suffers from very high popularity (Falci and McNeely 2009). The results here find that for depressive youth, this pattern remains visible for mental health years later.

Notably, these effects of status are only found for depressive teens. For those who were not experiencing depressive symptoms in adolescence, popularity and prestige do not predict young adult depressive levels. This finding suggests that depressive youth may be most at risk for long-term effects of lacking friends or stressfully high prestige. Alternatively, non-depressive adolescents may better adapt to young adult roles and environments so that adolescent peer positions have no lasting effect upon young adult mental health. Further research should investigate the mechanisms of resilience for non-depressive teens compared to risks for depressive teens from low and very high social status.

Second, peer network structural support interacts with adolescent friends' depressive symptoms. Greater cohesion among depressive friends predicts higher depressive symptoms in young adulthood (net of adolescents' own depressive levels). Cohesive integration among friends experiencing depressive symptoms may increase stressful demands for support, deter the development of positive skills and psychosocial benefits associated with healthy supportive friendships, or foster co-rumination behaviors that individuals carry into young adulthood (Rose et al. 2014; Cheadle and Goosby 2012). These results align with research indicating risks of network integration among depressive peers (Rose et al. 2014; Reynolds and Crea 2015). Findings here extend this work by indicating that risks of cohesive depressive friend groups can affect mental health after adolescence.

Unlike cohesion, reciprocity is non-significant. This pattern suggests the importance of considering larger patterns of network structure, such as how individuals' friends connect to each other, rather than isolated dyads or individual friendships.

This study has several limitations. Data come from respondents in semi-rural Iowa and Pennsylvania, so patterns may differ in less rural or more diverse settings. For example, less rural communities may have more schooling options in a geographic area that foster greater out-of-school connections to reduce the salience of in-school peers for long-term mental health, or more diverse student bodies may have greater heterogeneity in network structure and how that structure relates to mental health. Data also capture different measures at different life stages, necessitating modeling general averages rather than 
modeling within-person change in the same measures over time. Network data capture one snapshot of friendships once a year and are restricted to in-school, in-grade peers. As a result, teens that have friendships outside of their grade in school may appear more isolated in these data than they are in reality in ways that might skew associations between networks and long-term mental health, though control measures adjusting for reported number of out-of-grade friends are non-significant in all models. Network data also do not provide measures of relationship quality, content, or the qualitative experience of connections with peers, which may also affect long-term mental health. As a survey, if those with poor mental health are more likely to miss school (and consequently survey administration in adolescence) or drop out of the survey at follow up, results could present a biased picture of networks predicting mental health. Data here pre-date widespread digital technologies and social media that may have changed network processes or how networks relate to mental health for current teens. Finally, several control variables in young adulthood are measured contemporaneously with the outcome measure of depressive symptoms, so causal direction of these associations cannot be determined.

Future work should address the gaps left by these limitations by examining trajectories of networks and mental health among more representative samples to examine if patterns observed here extend beyond rural communities, and by examining how relationship quality and content relate to the processes delineated here. This study raises new questions of the mechanisms through which adolescent social position relates to subsequent health and how long such associations persist into adulthood, which should also be examined in future studies.

Despite limitations, these data and analyses still provide a unique and valuable opportunity to examine the research questions at hand. This work suggests researchers and practitioners engaging with adolescents should consider structures of status and support among peers as opportunities for enduring risks or benefits to mental health. For example, because high prestige positions can spur later depressive symptoms, those working with youth should remain attentive to risks of over-integration among peers, particularly for teens experiencing depressive symptoms. Researchers, practitioners, and parents should also consider the potential for long-term risks of both low status and very high-status positions among peers for youth experiencing depressive symptoms, and that belonging to a tight-knit group of depressive friends may be detrimental for mental health even after adolescence. In this way, considering how teens' and friends' mental health may interact with how teens are connected to peers can be helpful for understanding long-term risks to mental health.

This study illuminates the important role of adolescent peer networks in young adult mental health. Results indicate that positions of status and structural support in teen networks predict depressive symptoms in young adulthood, but such associations depend upon the mental health of adolescents and their friends. This study highlights the importance of considering network content and structure in tandem, as the association between networks structures and subsequent mental health varies with the mental distress of those connected in the network. This work also indicates the value of viewing social networks in the life course perspective, with adolescent network position as one salient feature in a sensitive period for development that can shape outcomes in subsequent life course stages. As such, this study provides a critical step in clarifying the association between adolescent peer networks and long-term mental health.

Funding: Analyses used data from PROSPER, funded by grant R01 DA013709 from the National Institute on Drug Abuse, and supported by R01 DA018225 from the National Institute on Drug Abuse.

Institutional Review Board Statement: Data collection for data used in this study met with ethical guidelines and approval from institutional review boards of managing institutions.

Informed Consent Statement: Informed consent was obtained from all subjects involved in the study.

Data Availability Statement: Due to data use agreements and to protect respondent privacy, data and code are not available to be shared for this project. 
Conflicts of Interest: The author declares no conflict of interest.

\section{Note}

1 Throughout, status refers to social status as in standing or position in the hierarchy among peers, rather than social characteristics such as gender or race/ethnicity.

\section{References}

Allen, Joseph P., Joanna Chango, David Szwedo, and Megan Schad. 2014. Long-Term Sequelae of Subclinical Depressive Symptoms in Early Adolescence. Development and Psychopathology 26: 171-80. [CrossRef]

Bonacich, Phillip. 1987. Power and Centrality: A Family of Measures. American Journal of Sociology 92: 1170. [CrossRef]

Cheadle, Jacob E., and Bridget J. Goosby. 2012. The Small-School Friendship Dynamics of Adolescent Depressive Symptoms. Society and Mental Health 2: 99-119. [CrossRef]

Cohen, Sheldon. 2004. Social Relationships and Health. American Psychologist 59: 676-84. [CrossRef]

Cook, Stephanie H., Justin E. Heinze, Alison L. Miller, and Marc A. Zimmerman. 2016. Transitions in Friendship Attachment during Adolescence Are Associated with Developmental Trajectories of Depression Through Adulthood. Journal of Adolescent Health 58: 260-66. [CrossRef]

Cornwell, Benjamin. 2003. The Dynamic Properties of Social Support: Decay, Growth, and Staticity, and Their Effects on Adolescent Depression. Social Forces 81: 953-78. [CrossRef]

Costello, Darcé M., Joel Swendsen, Jennifer S. Rose, and Lisa C. Dierker. 2008. Risk and Protective Factors Associated with Trajectories of Depressed Mood from Adolescence to Early Adulthood. Journal of Consulting and Clinical Psychology 76: 173-83. [CrossRef]

Cotterell, John. 2007. Social Networks in Youth E Adolescence. New York: Routledge.

Durkheim, Emile. 2006. On Suicide. London: Penguin. First published 1897.

Edgerton, Jason D., Souradet Shaw, and Lance W. Roberts. 2018. An Exploration of Depression Symptom Trajectories, and Their Predictors, in a Canadian Sample of Emerging Adults. Emerging Adulthood 7: 352-62. [CrossRef]

Falci, Christina, and Clea McNeely. 2009. Too Many Friends: Social Integration, Network Cohesion and Adolescent Depressive Symptoms. Social Forces 87: 2031-62. [CrossRef]

Gore, Susan, and Robert H. Aseltine Jr. 2003. Race and Ethnic Differences in Depressed Mood Following the Transition from High School. Journal of Health and Social Behavior 44: 370-89. [CrossRef]

Hankin, Benjamin L. 2006. Adolescent Depression: Description, Causes, and Interventions. Epilepsy and Behavior 8: 102-14. [CrossRef]

Johnson, Monica Kirkpatrick, Robert Crosnoe, and Glen H. Elder Jr. 2011. Insights on Adolescence from a Life Course Perspective. Journal of Research on Adolescence 21: 273-80. [CrossRef]

Kamis, Christina, and Molly Copeland. 2020. The Long Arm of Social Integration: Gender, Adolescent Social Networks, and Adult Depressive Symptoms. Journal of Health and Social Behavior 61: 437-52. [CrossRef]

Kornienko, Olga, and Carlos E. Santos. 2014. The Effects of Friendship Network Popularity on Depressive Symptoms During Early Adolescence: Moderation by Fear of Negative Evaluation and Gender. Journal of Youth and Adolescence 43: 541-53. [CrossRef] [PubMed]

Krause, Robert W., Mark Huisman, Christian Steglich, and Tom Snijders. 2020. Missing Data in Cross-Sectional Networks-An Extensive Comparison of Missing Data Treatment Methods. Social Networks 62: 99-112. [CrossRef]

McGrath, Emily P., and Rena L. Repetti. 2002. A Longitudinal Study of Children's Depressive Symptoms, Self-Perceptions, and Cognitive Distortions about the Self. Journal of Abnormal Psychology 111: 77-87. [CrossRef]

Millings, Abigail, Rhiannon Buck, Alan Montgomery, Melissa Spears, and Paul Stallard. 2012. School Connectedness, Peer Attachment, and Self-Esteem as Predictors of Adolescent Depression. Journal of Adolescence 35: 1061-67. [CrossRef]

Moody, James, Wendy D. Brynildsen, D. Wayne Osgood, Mark Feinberg, and Scott Gest. 2011. Popularity Trajectories and Substance Use in Early Adolescence. Social Networks 33: 101-12. [CrossRef] [PubMed]

Ohannessian, Christine McCauley, Anna Vannucci, Courtney R. Lincoln, Kaitlin M. Flannery, and Ashley Trinh. 2018. Self-Competence and Depressive Symptoms in Middle-Late Adolescence: Disentangling the Direction of Effect. Journal of Research on Adolescence 29: 1-16. [CrossRef] [PubMed]

Radloff, Lenore Sawyer. 1977. The CES-D Scale: A Self-Report Depression Scale for Research in the General Population. Applied.Psychological Measurement 1: 385-401. [CrossRef]

Reynolds, Andrew D., and Thomas M. Crea. 2015. Peer Influence Processes for Youth Delinquency and Depression. Journal of Adolescence 43: 83-95. [CrossRef]

Rose, Amanda J., Rebecca A. Schwartz-Mette, Gary C. Glick, Rhiannon L. Smith, and Aaron M. Luebbe. 2014. An Observational Study of Co-Rumination in Adolescent Friendships. Developmental Psychology 50: 2199-209. [CrossRef]

Schaefer, David R., Olga Kornienko, and Andrew M. Fox. 2011. Misery Does Not Love Company: Network Selection Mechanisms and Depression Homophily. American Sociological Review 76: 764-85. [CrossRef]

Segrin, Chris, and Kristina N. Rynes. 2009. The Mediating Role of Positive Relations with Others in Associations between Depressive Symptoms, Social Skills, and Perceived Stress. Journal of Research in Personality 43: 962-71. [CrossRef]

Smith, Jeffrey A., James Moody, and Jonathan H. Morgan. 2017. Network Sampling Coverage II: The Effect of Non-Random Missing Data on Network Measurement. Social Networks 48: 78-99. [CrossRef] [PubMed] 
Spoth, Richard, Cleve Redmond, Chungyeol Shin, Mark Greenberg, Mark Feinberg, and Lisa Schainker. 2013. PROSPER CommunityUniversity Partnership Delivery System Effects on Substance Misuse through 61/2 Years Past Baseline from a Cluster Randomized Controlled Intervention Trial. Preventive Medicine 56: 190-96. [CrossRef] [PubMed]

Spoth, Richard, Mark Greenberg, Karen Bierman, and Cleve Redmond. 2004. PROSPER Community-University Partnership Model for Public Education Systems: Capacity-Building for Evidence-Based, Competence-Building Prevention. Prevention Science 5: 31-39. [CrossRef]

Thoits, Peggy A. 2011. Mechanisms Linking Social Ties and Support to Physical and Mental Health. Journal of Health and Social Behavior 52: 145-61. [CrossRef]

Ueno, Koji. 2005. The Effects of Friendship Networks on Adolescent Depressive Symptoms. Social Science Research 34: 484-510. [CrossRef]

Umberson, Debra, Robert Crosnoe, and Corinne Reczek. 2010. Social Relationships and Health Behavior across Life Course. Annual Review of Sociology 36: 139-57. [CrossRef]

Valente, Thomas W. 2010. Social Networks and Health, 1st ed. Oxford: Oxford Press.

Wooldridge, Jeffrey M. 2012. Introductory Econometrics: A Modern Approach, 5th ed. Mason: Cengage Learning. 